19 Revue d'histoire du XIXe siècle

Société d'histoire de la révolution de 1848 et des

révolutions du XIXe siècle

22 | 2001

Autour de Décembre 1851

La Corse et la République, la vie politique de 1870 à 1914, Thèse pour le doctorat en histoire

Jean-Paul Pellegrinetti

OpenEdition

Journals

Édition électronique

URL : http://journals.openedition.org/rh19/290

DOI : $10.4000 /$ rh 19.290

ISSN : $1777-5329$

Éditeur

La Société de 1848

Édition imprimée

Date de publication : 1 juin 2001

ISSN : 1265-1354

Référence électronique

Jean-Paul Pellegrinetti, « La Corse et la République, la vie politique de 1870 à 1914, Thèse pour le doctorat en histoire », Revue d'histoire du XIXe siècle [En ligne], 22 | 2001, mis en ligne le 04 septembre 2008, consulté le 30 avril 2019. URL : http://journals.openedition.org/rh19/290; DOI : 10.4000/ rh19.290

Ce document a été généré automatiquement le 30 avril 2019

Tous droits réservés 


\title{
La Corse et la République, la vie politique de 1870 à 1914, Thèse pour le doctorat en histoire
}

\author{
Jean-Paul Pellegrinetti
}

1 Thèse pour le doctorat en histoire sous la direction de Ralph Schor, Université de NiceSophia-Antipolis, 4 volumes, $1148 \mathrm{f}^{\circ}$, soutenue en septembre 2000 devant un jury composé de Jacques Basso, Pierre Guillaume, Jean-Jacques Becker, Ralph Schor et Michel Winock, mention très honorable avec les félicitations du jury.

$\mathrm{Au}$ même titre que pour l'histoire nationale, les années allant de la chute du régime impérial à la Première Guerre mondiale occupent une place prépondérante dans l'histoire de la Corse. Elles constituent, en effet, une époque charnière correspondant à l'ancrage définitif de l'île dans la République. La Corse, à l'image d'autres départements, participe ainsi aux difficiles combats menés par les partisans de Marianne face aux conservateurs. L'île, présente, à la fin du XIXe siècle et durant les premières années du XXe siècle, des caractères spécifiques. Elle constitue, en effet, une terre où les marques de fidélité à la famille Bonaparte sont omniprésentes. La Corse, autre singularité, est une terre clanique et clientéliste. À la chute de Napoléon III, l'île continue ainsi, par le biais de réseaux de clientèles et de parentèles, à manifester jusqu'en 1878 son entier dévouement aux hommes et aux idées impérialistes. Elle est, de ce fait, le bastion de quelques grandes familles dont l'ascension professionnelle et politique effectuée durant le règne de l'empereur leur permet, après la défaite de Sedan, de maintenir en place leur hégémonique pouvoir politique. De 1870 à 1914, la société insulaire est essentiellement une société rurale dont l'économie repose sur une utilisation des sols répartie entre l'agriculture, l'arboriculture et le pastoralisme. À la fin du XIXe siècle, à la suite d'une grave crise agricole, l'économie insulaire connaît un effondrement. Différents secteurs de production sont touchés : le vignoble est phylloxéré; la céréaliculture est incapable de faire face à la concurrence des blés étrangers ; l'oléiculture et la production laitière enfin régressent fortement par manque de débouchés. L'activité industrielle, très 
embryonnaire et fragile, ne permet pas un redressement de la situation. À la fin du Second Empire, la Corse est, malgré quelques tentatives, largement restée à l'écart du capitalisme et de la révolution industrielle. L'expansion coloniale française dans le monde et l'amélioration des moyens de transport conduisent une partie de la population miséreuse à s'expatrier vers des contrées moins rudes et synonymes d'amélioration du statut social, compte tenu des postes proposés par la fonction publique. La crise économique accentuant les pratiques clientélistes anciennes, les élites politiques deviennent, pour les migrants, des intermédiaires obligés. Leurs rapports, en périodes électorales, se concrétisent souvent par l'échange d'un bulletin de vote contre celui d'une place.

La dernière des particularités demeure liée à la politique. Cette dernière est, en effet, vécue comme une véritable passion; elle est, pour les insulaires, synonyme de puissance, de pouvoir et d'honneur. Le jeu des urnes revêt lui aussi un sens particulier. La politique est avant tout une affaire de familles regroupées à l'intérieur de partis locaux qui se positionnent eux-mêmes au fil des scrutins derrière des personnalités éponymes. Pour la masse électorale, à la base de la structure pyramidale de la société, les liens qui l'unissent aux notabilités politiques semblent très éloignés des blasons partisans et idéologiques de leurs représentants. Le vote, compte tenu des multiples enjeux qu'il contient, est souvent à l'origine de conflits familiaux où la violence constitue fréquemment une pièce maitresse dans le règlement des rivalités.

Bastion bonapartiste, terre de clanisme, de clientélisme et de misère économique, la Corse apparait, du fait également de son insularité, comme éloignée de tous mouvements politiques nationaux et comme écartée complètement de tous débats idéologiques. Dans les dernières années du XIXe siècle, tout concourt à rendre ainsi fort précaire, l'existence de "Marianne " dans les communautés villageoises composantes de la société insulaire. Pourtant, dès 1878 , les républicains enregistrent leurs premières victoires électorales et l'idée républicaine gagne progressivement du terrain dans l'île. Fruit d'intenses luttes et combats politiques, la République s'installe de manière durable en Corse à partir de 1898.

L'étude de la République en Corse à travers le prisme de la vie politique entre 1870 et 1914, constitue un complément et un éclairage supplémentaire sur l'histoire générale de la Troisième République. Elle se veut pour cela répondre à cinq interrogations initiales : selon quels moyens, quelles structures, quelles formes, quel discours et enfin grâce à quels hommes, la République s'est-elle enracinée dans l'île? Traitée à partir d'un découpage chronologique en trois parties qui fait ressortir, de 1871 à 1877, l'ancrage bonapartiste de l'île, de 1878 à 1893, la conquête républicaine, et de 1894 à 1914, le triomphe de la République et ses mutations internes, l'analyse de la vie politique en Corse sous la Troisième République met en évidence les mécanismes et les pratiques politiques insulaires, les corrélations entre la politique au village et les amples respirations chronologiques qui affectent l'ensemble du pays. Elle propose également une typologie des structures formelles des organisations et des partis politiques dans leurs évolutions et leurs cheminements singuliers. Enfin, elle identifie dans l'espace, des frontières, mais également des zones de permanence et de mutation.

De 1870 à 1878, la Corse constitue un véritable bastion pour les anciennes notabilités d'Empire dont la puissance des réseaux clientélistes leur permet d'exercer une véritable hégémonie politique dans tout le département. Durant cette période, l'accès à la représentation politique repose uniquement sur une légitimité créée par le droit du sang. L'île apparaît comme ancrée à l'intérieur d'une domination sociale ancienne et traditionnelle et les échanges clientélistes semblent l'écarter de toutes formes de débats 
idéologiques. Seules de grandes lignées familiales dominent véritablement le débat politique. Leurs ressources, nécessaires dans les échanges avec la clientèle, demeurent essentiellement celles procurées par la propriété foncière.

Les années allant de 1878 à 1893, correspondent à la conquête républicaine. Sur la scène politique départementale, de nouvelles lignées partisanes viennent concurrencer les anciennes familles bonapartistes. L'arrivée, à l'échelon national, des républicains au pouvoir procure aux élites nouvelles insulaires le droit de prétendre à l'éligibilité. D'autres formes de ressources, liées à un investissement professionnel ou à une réussite universitaire, apparaissent. Dans l'île, de nouvelles pratiques politiques s'enracinent très lentement. La Corse adhère de manière progressive à la République, l'idée républicaine se propage ainsi à l'intérieur des communautés villageoises pour qui commence, également, un long apprentissage de la politique.

De 1893 à 1914, la Troisième République, ancrée définitivement, triomphe en Corse. La forme du régime ne suscite plus que quelques contestations mineures, ressenties dans l'île à la suite d'affaires ou de crises nationales. Toutefois, si la République devient le régime stable et durable de la France de la fin du XIXe siècle et des quatorze premières années du XXe siècle, elle provoque d'importants classements en profondeur au niveau des forces partisanes insulaires. Au sein des partis politiques, le débat idéologique s'installe, par l'intermédiaire des professions de foi notamment, sur la manière d'être et de se présenter à l'électorat en tant que républicain.

$\mathrm{Au}$ centre de la vie politique insulaire, les membres du parti républicain modéré sont les principaux artisans de l'ancrage définitif de la Corse dans la République. À partir de 1878, les républicains utilisent toutes formes de méthodes et d'instruments qui, comme sur le plan national, s'avèrent prépondérants pour la mise en place d'une République opportuniste. Dès 1871, l'île devient le terreau d'une floraison progressive de comités communaux électoraux nécessaires pour une première structuration de l'électorat et une diffusion naissante de l'idée républicaine. Des cercles, certes peu nombreux, attestent eux aussi des profondes volontés de politisation des espaces publics avec la création de structures politiques permanentes. L'organisation électorale s'impose tout au long de la période comme un phénomène de gauche. À l'échelle locale, d'autres pratiques politiques voient progressivement le jour dans l'île. Les républicains utilisent ainsi tous les registres de la politique festive nécessaires pour marquer véritablement les consciences collectives. La République apparaît ainsi aux communautés villageoises sous les traits multiples de la fête, des banquets et des "punchs" électoraux, mais également sous les visages de Marianne, différents selon les périodes d'installation des bustes dans les mairies. À l'échelle départementale, d'autres armes et stratégies facilitent l'enracinement de la République. Les républicains modérés, à l'image des forces bonapartistes, mettent en place de nombreux réseaux politiques pyramidaux dont les liens tissés autour de l'idée républicaine commune, se renforcent notamment avec l'utilisation de témoins lors des cérémonies de mariage ou de parrains lors des baptêmes.

Le train, l'école et la construction du réseau routier, deviennent synonymes d'une ère nouvelle de progrès offerte aux populations. Durant la période considérée, tous les apports, économiques, sociaux et matériels du nouveau régime sont mis en avant par la presse propagandiste. Celle-ci sert également de lien entre les différentes notabilités. Elle est un support nécessaire, à la venue des masses rurales dans les rangs de la République dont l'un de ses principaux agents est le préfet. De la chute du Second Empire à la Première Guerre mondiale, les préfets sont les personnages clefs de la vie politique locale qui servent d'intermédiaires entre les structures étatiques et les autorités représentatives 
du département. Au sein des communautés villageoises, leurs fonctions, fortement politisées, les conduisent à devenir les défenseurs des mesures gouvernementales. Ils y sont également, en périodes électorales, des agents électoraux actifs au service de la République et de ses candidats.

Puissante, structurée et mobilisatrice au cours des toutes premières années de la Troisième République, la droite corse subit, dès 1878 , les effets non escomptés de la crise du 16 mai 1877, mais également les succès enregistrés à l'échelon national par les forces de la République. À la recherche d'une restauration impériale et victimes de luttes intestines, les membres de la droite corse sont, entre 1886 et 1908, partiellement écartés de la scène politique départementale. Leurs retours éphémères en 1889 lors du mouvement boulangiste, puis en 1893 au cours du ralliement à la République témoignent cependant d'une survie des lignées de notables héritiers du régime impérial. Les élites de la droite insulaire apprennent ainsi à évoluer au sein de la société et n'hésitent pas à s'investir dans des domaines nécessaires à la construction de la notabilité et dans celle donnant accès aux chemins de la représentativité électorale. En 1892, les forces bonapartistes, conscientes d'un impossible retour du régime impérial et d'un ancrage profond des idées républicaines, se rallient dans leur ensemble à la République. La droite républicaine insulaire récupère, à partir de 1908, une position hégémonique sur la scène politique départementale.

De 1871 à 1914, de multiples facteurs contribuent à la politisation des masses rurales. Cette acculturation progressive des communautés villageoises à la politique est le fruit de différents paramètres véhiculés pour l'essentiel par les élites politiques. Par l'intermédiaire de l'appartenance des notabilités à des réseaux politiques nationaux, par leurs mariages avec des épouses originaires du continent, leurs études dans des universités parisiennes pour la plupart, leur participation à une presse partisane et enfin, leurs discours empreints de marques idéologiques, la politique pénètre progressivement dans l'univers quotidien des villages insulaires. La presse, les affiches, mais également toutes formes de brochures, photographies et statuaires participent progressivement à une prise de conscience de l'interpénétration des enjeux politiques nationaux et locaux.

Avec l'enracinement du mode de scrutin, le principe de l'hérédité dans la transmission du pouvoir politique se retrouve concurrencé par celui reposant sur une légitimité démocratique. Le combat pour l'accès à la représentativité devient nettement plus ouvert. L'exercice du suffrage " universel " implique ainsi des modifications dans les rouages des pratiques politiques insulaires. Le personnel élitaire, dans l'obligation de rassembler le plus grand nombre d'électeurs, se voit ainsi appelé à la tête de différentes structures de sociabilité, comme celles des comités. Avec l'enracinement de la Troisième République et l'acculturation des populations insulaires à des pratiques nouvelles de la politique, des formes nouvelles d'associations de solidarité, de défense sociale, mais aussi de contestations, apparaissent dans l'île dès la fin du XIXe siècle.

L'analyse de la vie politique en Corse met en évidence, au sein de la société insulaire, de 1870 à 1914, deux formes de mécanismes politiques qui relèvent à la fois de la modernité et de l'archaïsme. Les élites politiques, toutes tendances confondues, manipulent ainsi les registres des pratiques officieuses et officielles en juxtaposant à la fois le discours idéologique, l'assimilation de méthodes politiques nationales et la continuité du système clientéliste ancien. La Troisième République s'installe en officialisant et en légitimant un espace public, tout en conservant néanmoins un espace privé à la base, nécessaire à la logique de son développement.

De la chute du Second Empire à la Première Guerre mondiale, la période correspondant à 
l'enracinement de la République en Corse met en évidence deux sortes de républicains dans l'île. Les premiers, qui forment le camp de la gauche insulaire, se définissent héritiers de la Révolution française ; ils se présentent comme attachés à la laïcité de l'État, à la Nation, aux réformes et aux droits sociaux et à la non-personnification du pouvoir. Les plus intransigeants d'entre eux font preuve également d'un anticléricalisme virulent. Quant aux seconds, ils représentent les forces de la droite insulaire. À partir de 1893, date de leur ralliement au régime républicain, souvent plus de raison que de cœur, ils s'affirment respectueux du régime et de l'état de droit, mais n'hésitent pas à rappeler leurs valeurs fondées sur le respect de la religion, de l'ordre et de la propriété privée.

À partir de 1904, les républicains modérés, qui édulcorent leur programme politique, se rapprochent des forces de la droite insulaire. Ils en rejoignent même les rangs à la mort d'Emmanuel Arène, leur chef de file. Les radicaux, quant à eux, récupèrent l'héritage de la tradition de la gauche républicaine, qui se veut combattante et non conciliante. Néanmoins, en 1908, le drapeau de la République flotte au sein des deux principales forces politiques de l'île. Si la lutte continue sur la manière de se définir en tant que républicain, force est de constater que la République s'implante dans un milieu qui, au cours des premières années qui suivent la chute de Napoléon III, lui est particulièrement hostile. Dans les deux camps toutefois, l'attachement à la République s'apparente à un attachement à la Nation tout entière. En 1914, près de 40000 Corses partent au front défendre la Mère Patrie. Le prix de leurs sacrifices, de leurs douleurs et de leurs croyances dans les valeurs de la République transparaît sur l'ensemble des monuments aux morts érigés à partir de 1920 dans tous les villages insulaires.

\section{INDEX}

Mots-clés : Histoire politique, République, Politisation 